\title{
A Retrospective Study of Bimodal Benefits of Cochlear Implant Children
}

\author{
Hyekyoung Hwang ${ }^{1}$, Timothy Kim ${ }^{1}$, Leehwa Park ${ }^{2,3}$, Soo Hee Oh ${ }^{2,3}$ \\ ${ }^{1}$ H.H.K Hearing and Speech Care Center, Seoul, Korea \\ ${ }^{2}$ Department of Audiology and Speech Language Pathology, Hallym Univesity of Graduate Studies, Seoul, Korea \\ ${ }^{3}$ Center for Hearing and Speech Research, Hallym University of Graduate Studies, Seoul, Korea
}

Received: August 9, 2021

Revised: November 25, 2021

Accepted: December 1, 2021

Correspondence:

Soo Hee Oh, PhD

Department of Audiology and

Speech Language Pathology, Hallym

Univesity of Graduate Studies, 427

Yeoksam-ro, Gangnam-gu, Seoul

06197, Korea

Tel: $+82-70-8680-6933$

Fax: $+82-2-3453-6618$

E-mail:osh503@naver.com
Purpose: Bimodal children showed various improvements in speech, language, and music perception. The purpose of this study is to investigate bimodal benefits and bimodal hearing aid fitting of cochlear implant children with a retrospective chart review. Methods: A total of 44 charts of cochlear implant children were retrospectively reviewed in this study. Hearing thresholds, word recognition scores, and hearing aid fitting procedures were reviewed and summarized. Results: Bimodal children showed 4\%points improvements in word recognition scores and 30 40 gains across frequencies with hearing aids. For bimodal hearing aid fitting, all children used desired sensation level $v 5$ fitting formula with wide frequency bands. Real ear measurements were performed to match with targets and loudness balancing across ears was also conducted. Hearing thresholds with and without hearing aids were not correlated with word recognition scores in bimodal condition. Conclusion: Bimodal children showed improved hearing thresholds with hearing aids and little bimodal benefits in word recognition tests. Additional protocols including speech in noise tests, subjective questionnaires are recommended to evaluate bimodal benefits.

Key Words: Bimodal fitting, Bimodal hearing, Hearing aid fitting, Bimodal children.

\section{INTRODUCTION}

양이청각은 두영(head shadow)효과, 양이진압(binaural squelch)효과, 양이합산(binaural summation)효과(Brown \& Balkany, 2007)를 통해 소음하 말지각 및 방향분별 측면에 서 효과가 있는 것으로 알려져 있다(Akeroyd, 2006; Byrne \& Noble, 1998; Dawes et al., 2013). 인공와우와 보청기를 양 귀에 각각 착용하는 바이모달은 이러한 양이청각의 혜택 이 외에도 음향과 전기의 서로 다른 자극을 보완적으로 활용하 여 소음하 말지각과 음악지각 등을 향상시킨다(Ching et al., 2004; Dorman et al., 2008; Kong et al., 2005; Warren \& Dunbar, 2018). 한 예로 선행 연구(Brown \& Bacon 2009; Crew et al., 2015; Dorman et al., 2008; Kong \& Carlyon, 2007)에서 저주파수 음향자극인 기본주파수(fundamental frequency, F0)가 바이모달 사용자의 소음하 말지각과 음악지

(c) This is an Open Access article distributed under the terms of the Creative Commons Attribution Non-Commercial License (https://creativecommons.org/ licenses/by-nc/4.0) which permits unrestricted non-commercial use, distribution, and reproduction in any medium, provided the original work is properly cited.
각 향상에 기여함을 보고하였다.

인공와우 대상자의 청력 기준이 성인(Gifford et al., 2010) 과 아동(Carlson et al., 2015) 모두 확대되는 추세에서, Scherf and Arnold(2014)는 인공와우 착용 아동의 약 $26 \%$ 가 반대편 귀에 보청기를 착용하는 바이모달 사용자임을 보고하였다. 바이 모달 착용 아동도 성인과 마찬가지로 조용한 상황과 소음 상황 의 말지각, 언어발달, 방향성, 음악지각 등 다양한 측면에서 바 이모달 혜택을 보였다(Carlson et al., 2015; Lotfi et al., 2019; Moberly et al., 2016; Nilakantan et al., 2018; Shirvani et al., 2015). Lotfi et al.(2019)의 연구에서는 바이모달 착용 아동 이 소음하검사에서 검사 조건에 따라 약 2 3 dB의 향상을 보였 고, 또 다른 연구(Moberly et al., 2016)에서는 아동의 발달 시 기에 바이모달 착용은 편측 인공와우만 착용했을 때보다 음운인 식(phonetic awareness)의 향상을 초래하여, 아동의 어휘, 작 업기억 및 독해 능력의 향상으로 이어진다고 보고한 바 있다.

바이모달 결과에 영향을 미칠 수 있는 요인으로는 양 귀 간 주 파수 불일치, 보청기 착용 귀의 잔존청력, 바이모달 적합, 소리 크기의 균형 등이 있다(Veugen et al., 2016a). 이외에도 Yoon 
Table 1. Participants' description

\begin{tabular}{|c|c|c|c|c|c|c|}
\hline Subject & Sex & Age & CI ear & Age of initial HA fit & Age of implantation & $\mathrm{CI}$ \\
\hline 1 & $\mathrm{M}$ & 10 y $1 \mathrm{~m}$ & $\mathrm{R}$ & $>1 y$ & $1 \mathrm{y} 4 \mathrm{~m}$ & Nucleus5/Cochlear \\
\hline 2 & $\mathrm{~F}$ & 8 y $6 \mathrm{~m}$ & $\mathrm{R}$ & $>1 y$ & 3 y $6 \mathrm{~m}$ & Sonet II/Medel \\
\hline 3 & M & 13 y $1 \mathrm{~m}$ & $\mathrm{R}$ & $>1 y$ & 5 y 2 m & Clarion/Advanced Bionics (AB) \\
\hline 4 & M & 16 y $1 \mathrm{~m}$ & $\mathrm{R}$ & $>1 y$ & 5 y $10 \mathrm{~m}$ & Freedom/Cochlear \\
\hline 5 & M & 11 y $8 \mathrm{~m}$ & $\mathrm{R}$ & $>1 y$ & $7 \mathrm{y} 1 \mathrm{~m}$ & Nucleus6/Cochlear \\
\hline 6 & M & 9 y $11 \mathrm{~m}$ & $\mathrm{~L}$ & $>1 y$ & $6 y$ & Rondo/Meldel \\
\hline 7 & $\mathrm{~F}$ & $8 y$ & $\mathrm{R}$ & $>1 y$ & $1 \mathrm{y}$ & Nucleus5/Cochlear \\
\hline 8 & M & 11 y $4 \mathrm{~m}$ & $\mathrm{~L}$ & $>1 y$ & 1 y $9 \mathrm{~m}$ & Nucleus5/Cochlear \\
\hline 9 & $\mathrm{~F}$ & $14 \mathrm{y} 4 \mathrm{~m}$ & $\mathrm{~L}$ & $>1 \mathrm{y}$ & 4 y $11 \mathrm{~m}$ & Freedom/Cochlear \\
\hline 10 & $\mathrm{~F}$ & 13 y $2 \mathrm{~m}$ & $\mathrm{R}$ & $>1 y$ & 2 y $11 \mathrm{~m}$ & Nucleus6/Cochlear \\
\hline 11 & M & 10 y $3 \mathrm{~m}$ & $\mathrm{R}$ & $>1 y$ & $3 y 10 \mathrm{~m}$ & Nucleus6/Cochlear \\
\hline 12 & $\mathrm{~F}$ & 14 y $8 \mathrm{~m}$ & $\mathrm{~L}$ & $>1 y$ & 2 y $5 \mathrm{~m}$ & Freedom/Cochlear \\
\hline 13 & M & 8 y $1 \mathrm{~m}$ & $\mathrm{R}$ & $>1 \mathrm{y}$ & 3 y $11 \mathrm{~m}$ & Kanso/Cochlear \\
\hline 14 & M & 15 y $3 \mathrm{~m}$ & $\mathrm{R}$ & $>1 y$ & 3 y $11 \mathrm{~m}$ & Freedom/Cochlear \\
\hline 15 & M & 16 y $8 \mathrm{~m}$ & $\mathrm{~L}$ & $>1 y$ & 6 y $3 \mathrm{~m}$ & Freedom/Cochlear \\
\hline 16 & M & 14 y $1 \mathrm{~m}$ & $\mathrm{R}$ & $>1 y$ & 2 y $3 \mathrm{~m}$ & Nucleus6/Cochlear \\
\hline 17 & M & 9 y $1 \mathrm{~m}$ & $\mathrm{R}$ & $>1 y$ & 1 y $7 \mathrm{~m}$ & Nucleus6/Cochlear \\
\hline 18 & $\mathrm{~F}$ & 10 y $1 \mathrm{~m}$ & $\mathrm{R}$ & $>1 y$ & $5 y$ & Nucleus6/Cochlear \\
\hline 19 & M & 15 y $8 \mathrm{~m}$ & $\mathrm{R}$ & $>1 y$ & 3 y $2 \mathrm{~m}$ & Freedom/Cochlear \\
\hline 20 & $\mathrm{~F}$ & 13 y $1 \mathrm{~m}$ & $\mathrm{R}$ & $>1 y$ & $2 \mathrm{y} 4 \mathrm{~m}$ & Nucleus7/Cochlear \\
\hline 21 & M & $14 \mathrm{y}$ & $\mathrm{R}$ & $>1 y$ & $4 \mathrm{~m}$ & Freedom/Cochlear \\
\hline 22 & M & 8 y $11 \mathrm{~m}$ & $\mathrm{~L}$ & $>1 y$ & 2 y $2 \mathrm{~m}$ & No information \\
\hline 23 & $\mathrm{~F}$ & 9 y $11 \mathrm{~m}$ & $\mathrm{~L}$ & $>1 \mathrm{y}$ & $5 y$ & Nucleus6/Cochlear \\
\hline 24 & M & 10 y $3 \mathrm{~m}$ & $\mathrm{R}$ & $>1 y$ & 4 y $4 \mathrm{~m}$ & Opus/Medel \\
\hline 25 & M & 6 y $8 \mathrm{~m}$ & $\mathrm{~L}$ & $>1 y$ & 5 y $3 \mathrm{~m}$ & Nucleus5/Cochlear \\
\hline 26 & $\mathrm{~F}$ & 11 y $7 \mathrm{~m}$ & $\mathrm{R}$ & $>1 \mathrm{y}$ & $3 y$ & Nucleus6/Cochlear \\
\hline 27 & M & 18 y $3 \mathrm{~m}$ & $\mathrm{~L}$ & $>1 y$ & 2 y $11 \mathrm{~m}$ & ESPrit3G/Cochlear \\
\hline 28 & $\mathrm{~F}$ & 15 y $4 \mathrm{~m}$ & $\mathrm{~L}$ & $>1 y$ & $5 y$ & Freedom/Cochlear \\
\hline 29 & M & 12 y $9 \mathrm{~m}$ & $\mathrm{~L}$ & $>1 y$ & $6 y$ & No information \\
\hline 30 & $\mathrm{~F}$ & 11 y $8 \mathrm{~m}$ & $\mathrm{R}$ & $>1 y$ & $2 \mathrm{y} 8 \mathrm{~m}$ & Kanso/Cochlear \\
\hline 31 & M & $7 y 6 \mathrm{~m}$ & $\mathrm{~L}$ & $>1 y$ & 2 y $8 \mathrm{~m}$ & Nucleus7/Cochlear \\
\hline 32 & $\mathrm{~F}$ & 22 y $4 \mathrm{~m}$ & $\mathrm{~L}$ & $>1 y$ & $13 y$ & Nucleus5/Cochlear \\
\hline 33 & M & $9 y$ & $\mathrm{R}$ & $>1 y$ & 3 y $3 \mathrm{~m}$ & Nucleus6/Cochlear \\
\hline 34 & M & 11 y $1 \mathrm{~m}$ & $\mathrm{R}$ & $>1 y$ & 3 y $5 \mathrm{~m}$ & Nucleus5/Cochlear \\
\hline 35 & M & $14 \mathrm{y} 1 \mathrm{~m}$ & $\mathrm{R}$ & $>1 y$ & 3 y $4 \mathrm{~m}$ & Freedom/Cochlear \\
\hline 36 & $\mathrm{~F}$ & $6 y 6 \mathrm{~m}$ & $\mathrm{~L}$ & $>1 y$ & 2 y $11 \mathrm{~m}$ & Medel \\
\hline 37 & M & $17 y$ & $\mathrm{R}$ & $>1 \mathrm{y}$ & 2 y $2 \mathrm{~m}$ & Nucleus5/Cochlear \\
\hline 38 & $\mathrm{~F}$ & 9 y $2 \mathrm{~m}$ & $\mathrm{~L}$ & $>1 y$ & 5 y $3 \mathrm{~m}$ & Rondo/Meldel \\
\hline 39 & M & 10 y $3 \mathrm{~m}$ & $\mathrm{R}$ & $>1 y$ & $3 y$ & Nucleus5/Cochlear \\
\hline 40 & $\mathrm{~F}$ & $9 y$ & $\mathrm{R}$ & $>1 y$ & - & Medel \\
\hline 41 & $\mathrm{~F}$ & $12 y$ & $\mathrm{R}$ & $>1 y$ & $3 y$ & Cochlear \\
\hline 42 & $\mathrm{~F}$ & 14 y $11 \mathrm{~m}$ & $\mathrm{R}$ & $>1 y$ & 3 y $10 \mathrm{~m}$ & Freedom/Cochlear \\
\hline 43 & M & 12 y $1 \mathrm{~m}$ & $\mathrm{R}$ & $>1 y$ & 5 y $9 \mathrm{~m}$ & Nucleus5/Cochlear \\
\hline 44 & M & 11 y $1 \mathrm{~m}$ & $\mathrm{~L}$ & $>3 y$ & 1 y $5 \mathrm{~m}$ & Freedom/Cochlear \\
\hline
\end{tabular}

CI: cochlear implant, HA: hearing aid, M: male, y: years, m: months, R: right, F: female, L: left 
et al.(2015)은 양 귀 이형기기를 통해 입력된 정보의 효율적인 통합 능력이 바이모달 결과 향상에 영향을 미칠 수 있음을 언급 하였다. 바이모달 적합은 바이모달 결과 향상에 영향을 미칠 수 있는 중요한 요인이며(Vroegop et al., 2019), 최근 문헌 연구 (Vroegop et al., 2018)에서는 보청기적합공식, 이형기기 간 음 량평형, 자동이득제어 조건, 주파수 밴드, 주파수하강시스템의 적용 등 바이모달 보청기적합 관련 연구를 정리하였다. 바이모달 보청기적합에서는 이렇듯 여러 사항에 대한 고려가 필요하나, 실 제 임상에서 바이모달 적합은 주로 인공와우 조절과 관리에 중 점을 두기 쉽고 보청기적합을 위한 노력과 전문적 관리가 제대로 제공되지 않을 수 있다(Park \& Oh, 2021; Siburt \& Holmes, 2015).

특히 아동의 바이모달 착용 결과는 바이모달 또는 양이 인공 와우 중 가장 효과적인 중재를 결정하는 중요한 근거가 될 수 있 다. 때문에 최적의 바이모달 적합과 관리 및 평가가 선행되어야 하며 이를 근거로 아동의 중재 방법을 결정하는 것이 바람직할 것이다. 아직까지 바이모달 착용 아동의 보청기적합에 대한 연구 는 많이 보고되지 않았으며, 바이모달 아동의 결과 향상에 도움 이 될 수 있는 바이모달 보청기적합 관련 연구가 좀 더 이루어져 야 할 것으로 본다.

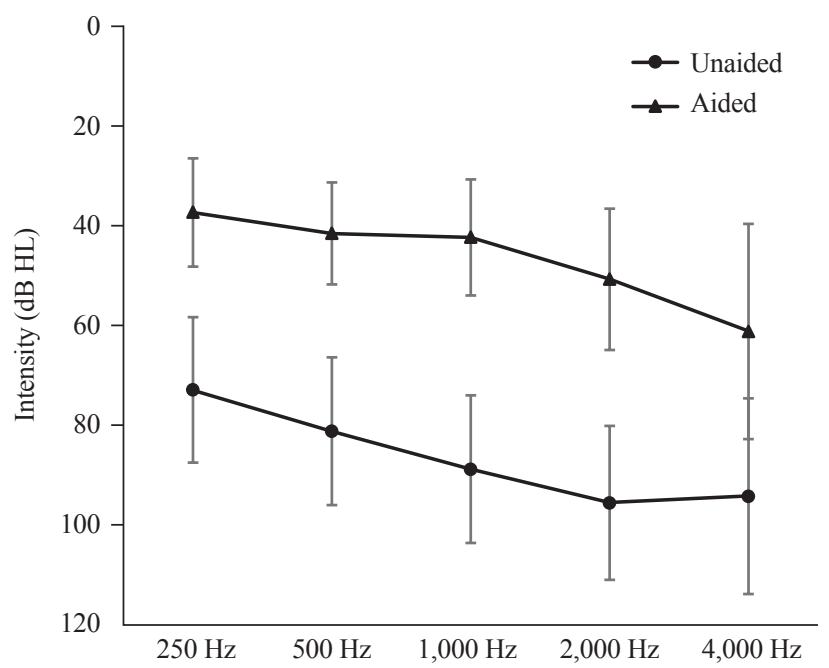

Figure 1. Aided and unaided hearing thresholds for the nonimplanted ear of bimodal children.
본 연구는 후향적 차트분석을 통해 아동의 바이모달 착용 효 과를 파악하는 데 목적이 있다. 청력정도, 기능이득, 보청기적합, 단어인지도 결과를 바탕으로 바이모달 착용 아동의 청력 및 말 지각 결과와 보청기적합의 전반적 내용을 분석해 보고자 한다. 연구 결과를 통해 바이모달 착용 아동의 보청기 착용 효과와 보 청기 적합에 대한 이해를 높이고 아동을 위한 바이모달 적합과 평가 프로토콜 기반 마련에 도움이 되고자 한다.

\section{MATERIALS AND METHODS}

\section{연구 방법}

2013년부터 2020년까지 HHK 청각언어센터를 방문한 44명 바이모달 착용 아동의 기록을 후향 분석하였다. 아동의 평균 나 이는 약 12.4세(약 8 22세)였으며 남자 27명, 여자 17명이었다. Table 1은 연구 대상자 정보를 요약한 내용이다. 인공와우 이 식 연령은 평균 약 3.8세(4개월 7세)로 대상자의 80\% (35명)는 Cochlear $^{\circledR}$ (Cochlear Limited, Sydney, Australia)사의 기기 를 착용하고 있었다. 대상자 아동 모두 언어습득 이전에 난청을 진단받았으며 난청을 늦게 발견하여 30개월에 보청기를 처음 착 용한 한 명을 제외하고 처음 보청기 착용은 1 세 이전에 이루어졌 다.

센터에서 시행한 바이모달 아동의 순음청력평가, 기능이득평 가, 단어인지도평가 및 보청기적합 결과를 분석하였고 모든 검 사는 방음실에서 진행하였다. 순음청력평가는 GSI Audiostar pro (Grason-Stadler INC., Eden Prairie, MN, USA)를 사 용하였다. $250 \mathrm{~Hz}$ 에서 $6,000 \mathrm{~Hz}$ 의 주파수 범위에서 $\mathrm{ER} 3 \mathrm{~A}$ insert earphone ( $3 \mathrm{M}^{\mathrm{TM}} \mathrm{E}-\mathrm{A}-\mathrm{RTONE}^{\mathrm{TM}}$, Saint Paul, MN, $\mathrm{USA}$ )을 착용한 상태에서 청력검사를 시행하여 각 주파수별 역치를 측정하였다. 기능이득평가는 $1 \mathrm{~m}$ 거리 정면에 위치 한 JBL Control 1Xtreme 스피커(Harman International Industries, Inc., Stamford, CT, USA)를 통해 와블톤을 제시 한 뒤 보청기와 인공와우를 착용한 조건에서 역치를 측정하였 다. 단어인지도검사는 보청기만 착용한 경우, 인공와우만 착용한 경우, 바이모달의 세 가지 조건에서 각각 시행하였고, $1 \mathrm{~m}$ 정면 음장에서 약 $65 \mathrm{~dB}$ SPL의 강도로 검사자가 일반, 학령기, 학령 전기의 한국표준단음절어(Korean Standard - Monosyllabic

Table 2. Mean functional gains for the non-implanted ear of bimodal children

\begin{tabular}{lccccc}
\hline Frequencies & $250 \mathrm{~Hz}$ & $500 \mathrm{~Hz}$ & $1,000 \mathrm{~Hz}$ & $2,000 \mathrm{~Hz}$ & $4,000 \mathrm{~Hz}$ \\
\hline Unaided mean thresholds (dB HL) & $73 \pm 15$ & $81 \pm 15$ & $89 \pm 15$ & $96 \pm 15$ & $94 \pm 20$ \\
Aided mean thresholds (dB HL) & $37 \pm 11$ & $42 \pm 10$ & $42 \pm 12$ & $51 \pm 14$ & $61 \pm 22$ \\
Mean functional gains (dB) & $36 \pm 15$ & $40 \pm 13$ & $46 \pm 11$ & $45 \pm 13$ & $32 \pm 14$ \\
\hline
\end{tabular}

Values are presented as mean \pm standard deviation 
Word Lists)를 육성으로 불러준 뒤 대상자가 따라 말하도록 하 였다. 보청기적합과 확인은 대상 아동의 청력을 근거로 각 보청 기 제조사의 보청기적합 소프트웨어와 Verifit2 ${ }^{\circledR}$ (Audioscan, Dorchester, Canada) 시스템을 활용하였다. 본 연구는 한림국 제대학원대학교 생명윤리심의위원회의 승인을 받아 시행하였다 (HUGSAUD509467).

\section{통계 분석}

각 조건별 단어인지도 값에 대한 정규성 검정(ShapiroWilk's)을 시행하였고 정규 분포되어 있지 않아 $(p<0.05)$ Friedman과 Wilcoxon Signed-Rank 검정을 시행하였다. 상 관관계 분석은 Spearman Rank-Order Correlation을 시행 하였다. SPSS (version 12.0; IBM Corp., Armonk, NY, USA) 를 사용하여 유의수준 0.05 이하를 기준으로 분석하였다.

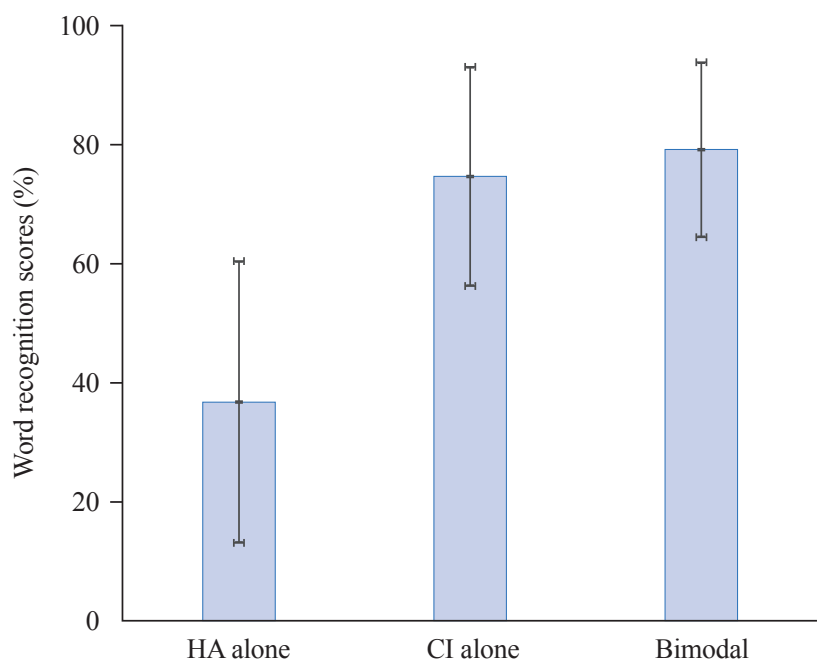

Figure 2. Word recognition scores of bimodal children across three test conditions. $\mathrm{HA}$ : hearing aid, $\mathrm{Cl}$ : cochlear implant.

\section{RESULTS}

\section{보청기 착용 귀의 청력역치와 기능이득}

44 명 바이모달 착용 아동의 보청기 착용 전과 후의 역치를 분 석한 결과 보청기 착용 전 평균 청력역치는 $250 \mathrm{~Hz}$ 에서 4,000 $\mathrm{Hz}$ 까지 $73 \mathrm{~dB} \mathrm{HL}$ 에서 $94 \mathrm{~dB} \mathrm{HL}$ 를, 보청기 착용 후 역치는 37 $\mathrm{dB}$ HL에서 $61 \mathrm{~dB}$ HL의 평균 역치를 나타냈다(Figure 1). 보 청기 착용 전 청력과 비교하였을 때 $250 \mathrm{~Hz}$ 에서 $4,000 \mathrm{~Hz}$ 까 지 전 주파수에서 보청기 착용 후 역치가 향상되었다 $(p<0.05$, Wilcoxon Signed-Rank test). 주파수별 기능이득은 $250 \mathrm{~Hz}$ 에서 $4,000 \mathrm{~Hz}$ 사이에 $33 \mathrm{~dB}$ 에서 $46 \mathrm{~dB}$ 를 보였다(Table 2).

\section{바이모달 착용 아동의 단어인지도}

바이모달 착용 아동의 단어인지도 평균은 보청기만 착용하였 을 때 $36.7 \%$, 인공와우만 착용하였을 때 $74.7 \%$, 바이모달 조건 에서는 $79.2 \%$ 로 각 검사 조건에서 의미 있는 차이를 보였고 $(p<$ 0.01, Friedman test), 인공와우만 착용하였을 때보다 바이모 달 착용시 약 $4 \%$ points의 향상을 보였다 $(p<0.01$, Wilcoxon Signed-Rank test) (Figure 2).

보청기 착용 귀의 청력 정도와 바이모달 단어인지도 결과와 의 상관성을 파악하기 위하여, 보청기를 착용한 상태와 착용하 지 않은 상태의 주파수별 청력 각각에 대해 보청기 또는 바이모 달 착용 상태에서의 단어인지도와의 상관관계를 분석하였다. 보 청기 착용 전과 후의 주파수별 청력 역치는 보청기만 착용하였 을 때의 단어인지도와 음의 상관관계를 보였으나(Spearman Rank-Order correlation coefficient, $p<0.05$ ) (Table 3), 바이모달 단어인지도와는 의미 있는 상관관계를 보이지 않았다 (Spearman Rank-Order correlation coefficient, $p>0.05$ ) (Table 3). 다시 말해, 보청기 착용 전과 후의 청력역치가 낮을수 록 보청기만 착용하였을 때 단어인지도의 향상과 상관관계가 있 으나 바이모달 단어인지도의 향상과는 관계가 없음을 알 수 있 었다.

Table 3. Correlation between unaided and aided thresholds and WRS scores

\begin{tabular}{|c|c|c|c|c|c|}
\hline Frequencies & $250 \mathrm{~Hz}$ & $500 \mathrm{~Hz}$ & $1,000 \mathrm{~Hz}$ & $2,000 \mathrm{~Hz}$ & $4,000 \mathrm{~Hz}$ \\
\hline \multicolumn{6}{|c|}{$\begin{array}{l}\text { Correlation between unaided thresholds and WRS scores } \\
\text { across frequencies }\end{array}$} \\
\hline HA WRS scores & $-5.12^{*}$ & $-6.03^{*}$ & $-6.65^{*}$ & $-5.86^{*}$ & $-4.67^{\star}$ \\
\hline Bimodal WRS scores & 0.11 & 0.05 & -0.06 & -0.07 & -0.08 \\
\hline \multicolumn{6}{|c|}{$\begin{array}{l}\text { Correlation between aided thresholds and WRS scores across } \\
\text { frequencies }\end{array}$} \\
\hline HA WRS scores & $-3.97^{\star}$ & $-3.96^{*}$ & $-4.95^{*}$ & $-4.25^{\star}$ & $-3.76^{*}$ \\
\hline Bimodal WRS scores & 0.04 & 0.01 & -0.05 & 0.02 & 0.11 \\
\hline
\end{tabular}

${ }^{*} p<0.05$. WRS: word recognition score, HA: hearing aid 
Table 4. Hearing aid information of the non-implanted ear of bimodal children

\begin{tabular}{|c|c|c|c|c|}
\hline Subject & Lower frequency $(\mathrm{Hz})$ & Upper frequency $(\mathrm{Hz})$ & HFA OSPL90 (dB SPL) & HA model \\
\hline 1 & 200 & 2,800 & 122 & Naida V BTE \\
\hline 2 & 200 & 7,100 & 106 & Naida Q90 BTE \\
\hline 3 & 200 & 4,240 & 109 & Naida Q50 BTE \\
\hline 4 & 235 & 6,300 & 116 & Boost17 BTE \\
\hline 5 & 200 & 3,775 & 112 & Legend9 ITC \\
\hline 6 & 375 & 6,725 & 112 & Legend9 ITC \\
\hline 7 & 200 & 2,500 & 119 & Boost9 BTE \\
\hline 8 & 200 & 7,550 & 111 & Legend17 CIC \\
\hline 9 & 200 & 4,500 & 116 & Ups7 BTE \\
\hline 10 & 200 & 7,100 & 113 & Trust9 ITE \\
\hline 11 & 200 & 4,500 & 125 & UPS9 BTE \\
\hline 12 & 200 & 2,800 & 116 & UPS9 BTE \\
\hline 13 & 200 & 6,300 & 110 & Aquaris3 mi BTE \\
\hline 14 & 300 & 2,670 & 122 & Nauda S lll BTE \\
\hline 15 & 200 & 7,100 & 109 & Legend9 CIC \\
\hline 16 & 200 & 7,100 & 105 & Legend9 CIC \\
\hline 17 & 200 & 5,000 & 133 & Sky B50 BTE \\
\hline 18 & 200 & 4,500 & 109 & Legend9 ITC \\
\hline 19 & 200 & 3,550 & 121 & Naida S V BTE \\
\hline 20 & 200 & 4,500 & 110 & Ambra CIC \\
\hline 21 & 280 & 4,240 & 125 & Sky Q50 BTE \\
\hline 22 & 200 & 5,600 & 122 & Naida Q50 BTE \\
\hline 23 & 200 & 6,725 & 107 & Legend9 ITC \\
\hline 24 & 355 & 5,340 & 113 & Naida Q50 BTE \\
\hline 25 & 200 & 3,775 & 116 & Naida Q570 BTE \\
\hline 26 & 200 & 4,500 & 118 & Legand17 ITC \\
\hline 27 & 200 & 4,000 & 117 & Sky V90 BTE \\
\hline 28 & 200 & 4,760 & 107 & Virto Q70 ITC \\
\hline 29 & 200 & 2,800 & 110 & Naida lll BTE \\
\hline 30 & 200 & 3,775 & 117 & Legend9 ITC \\
\hline 31 & 500 & 5,000 & 118 & Legend9 ITC \\
\hline 32 & 200 & 7,100 & 114 & Legend17 CIC \\
\hline 33 & 200 & 7,550 & 110 & Legend9 ITC \\
\hline 34 & 200 & 4,760 & 117 & Legend9 ITC \\
\hline 35 & 200 & 4,760 & 111 & Naida Q50 BTE \\
\hline 36 & 200 & 3,550 & 109 & Naida S IX BTE \\
\hline 37 & 200 & 6,000 & 115 & Naida lll BTE \\
\hline 38 & 200 & 4,000 & 106 & Legend9 ITC \\
\hline 39 & 200 & 3,775 & 127 & Ups7 BTE \\
\hline 40 & 200 & 7,550 & 109 & LND6 ITC \\
\hline 41 & 225 & 3,365 & 118 & Xceed III BTE \\
\hline 42 & 200 & 4,500 & 117 & Legend6 ITC \\
\hline 43 & 200 & 5,000 & 107 & Sky B50 BTE \\
\hline 44 & 200 & 4,500 & 110 & PSE9 ITC \\
\hline
\end{tabular}

HFA OSPL90: high frequency average output sound pressure level 90, HA: hearing aid, BTE: behind the ear, ITC: in the ear, CIC: complete in the canal 


\section{바이모달 아동의 보청기적합 내용 분석}

Table 4는 44명 바이모달 착용 아동이 착용하고 있는 보청 기 모델과 관련 정보를 보여준다. 아동의 $55 \%$ 는 귀걸이형 보청 기(behind the ear)를 착용하고 있었고, 34\%는 귓속보청기(in the ear), $11 \%$ 는 고막보청기(completely in the canal)를 착 용하고 있었다. 보청기 주파수 범위(frequency range)는 평균 $220( \pm 58.2) \sim 4,944( \pm 1,497) \mathrm{Hz}$ 였으며, 최저 주파수와 최 고 주파수 범위는 200 7,550 Hz였다. 고주파수 평균 OSPL90 (high frequency average output sound pressure level 90, HFA OSPL90)값의 평균은 114 ( \pm 6.3) dB SPL로 나타났다. 44명 아동 모두 desired sensation level v5 (DSL v5) 보청기적합공 식으로 적합하였다(Bagatto et al., 2005).

보청기적합은 Noahlink를 활용하여 각 제조사의 보청기 적합 소프트웨어를 통해 조절하면서 Verifit2 ${ }^{\circledR}$ (Audioscan) 에서 제시한 연령별 목표이득에 매치하도록 하였다. Verifit $2^{\circledR}$ (Audioscan)의 speechmap을 활용하여 작은 소리(50 dB $\mathrm{SPL})$, 보통 소리(65 dB SPL), 큰 소리(80 dB SPL)의 입력 말소 리(International Speech Test Signal) (Holube et al., 2010) 를 제시한 상태에서 주파수 실이증폭반응(real-ear aided response)과 최대출력(maximum power output) 및 Speech Intelligibility Index (SII)를 확인하면서 목표이득 값에 매치하 도록 보청기 이득을 조절하였다. 또한 대상자에게 말소리를 들려 주고 보청기 착용 귀의 소리크기를 반대편 인공와우 착용 귀의 소리크기와 비교하도록 하였다. 이때 인공와우 착용 귀에서 들리 는 소리보다 보청기를 착용한 귀의 소리가 약간 작으면서도 편안 하면서도 균형을 이루는 소리크기 범위 내에서 보청기를 조절하 였다.

\section{DISCUSSIONS}

본 연구에서는 후향적 챠트 분석을 통해 바이모달 아동의 보 청기 착용 후 청력역치 및 기능이득, 단어인지도검사 결과와 바 이모달 보청기적합의 내용을 확인하였다. 먼저 연구 결과에서 보여준 바이모달 혜택은 평균 약 $4 \%$ points로 다소 작았다 $(p<$ 0.05). Cheng et al.(2018)의 연구에서는 바이모달 사용자를 대 상으로 조용한 상황의 모음, 자음, 문장 검사를 시행한 결과 각 각 평균 $2 \%$ points, $7 \%$ points, $3 \%$ points의 통계적으로 유의 하지 않은 $(p>0.05)$ 향상을 보고한 바 있으며 이를 천장효과 (ceiling effect)로 설명하였다. 본 연구에서도 인공와우만 착용 하였을 때 단어인지도검사 결과는 평균 $75 \%$, 바이모달 조건에서 는 79\%를 보여 상당히 높은 점수를 보였다. 이러한 천장효과의 한계를 극복하고 바이모달 효과를 측정하기 위해서는 다른 선행 연구(Brown \& Bacon, 2009; Crew et al., 2015)처럼 소음하
말지각검사의 시행이 필요할 것으로 생각된다.

바이모달 착용효과는 인공와우 신호처리에서 주로 전달되 는 말소리의 포락선(envelope) 단서 외에 인공와우의 전기적 자극에 포함되지 않은 기본주파수(F0), 말소리 미세정보(fine structure) 등의 저주파수 음향 자극을 보완 단서로 활용하는 것과 관련이 있는 것으로 알려져 있다(Ching et al., 2004). 양 귀 각각의 보완적 음향 단서와 인공와우의 전기적 단서는 통합, 해석되어 말지각 향상에 도움이 된다.

바이모달 보청기적합 관련 선행 연구에서는 실이측정과 목표 이득에 부합하는 보청기적합의 중요성을 언급하였다(Harris \& Hay-McCutcheon, 2010; Yehudai et al., 2013). 본 연구에 서도 실이측정을 시행하여 목표이득에 부합하는 바이모달 보청 기적합을 시행하였고 이는 바이모달 아동의 결과 향상에 영향 을 미치는 요인으로 작용하였을 것으로 생각된다. 또한 아동 모 두 DSL v5 적합공식을 적용하였는데 선행 연구(Cuda et al., 2019; English et al., 2016)에서는 보청기적합공식에 따른 결 과의 차이는 크게 보고되지 않아 DSL v5 공식의 적용이 바이모 달 혜택에 부정적 영향을 미쳤을 것으로 생각되지는 않는다.

보청기 주파수 밴드의 설정에서 와우사 영역(Moore \& Malicka, 2013)을 고려하지는 않았으며 대부분 아동의 잔존 청력을 근거로 $4,000 \mathrm{~Hz}$ 까지 보청기적합을 시행하였다. 최근 D'Onofrio and Gifford(2021)의 보고에서도 보청기 주파수 밴 드를 확장한 경우 말지각과 음악지각이 향상되었음을 보고하였 다. 와우사 영역 등 다른 이유가 없다면 주파수 밴드의 확장을 기본 보청기적합으로 적용하여 보청기를 조절하는 것이 적절할 것으로 생각된다. 와우사 영역을 고려한 주파수 설정이 결과 향 상에 기여할 수 있기 때문에 바이모달 착용 아동의 경우에도 와 우사 영역을 고려한 주파수 설정이 적용될 수 있다면 좀 더 최적 화된 바이모달 보청기적합에 근접할 수 있을 것이다(Zhang et al., 2014). 또한 아동의 주파수별 잔존청력 또는 보청기 착용 후 청력이 바이모달 말지각검사 결과와 의미 있는 상관관계는 보이 지 않는 것으로 나타났다. Yoon et al.(2015)의 연구에서도 이와 비슷한 결과를 보였으며 바이모달 말지각 향상에 영향을 미치는 요인으로 이형기기 간 자극을 효과적으로 통합하는 능력과 관계 가 있을 수 있음을 설명하였다.

마지막으로 여러 선행 연구에서 양이 간 소리크기의 균형을 평가하고 적절한 균형을 유지하는 것의 중요성을 보고하였다 (Ching et al., 2004; Keilmann et al., 2009; Veugen et al., 2016b). 임상에서 양 귀 간 소리크기의 균형을 평가하고 조절하 기 위한 지침은 아직까지 미비하며, 주관적 판단에 의해 소리크 기를 평가하는 것이기 때문에 실제 검사를 수행하는 데 어려움 이 따를 수 있다. 또한 소리크기에 대한 판단 근거가 대부분 인공 와우 소리크기를 기준으로 하고 있으며 소리크기의 균형을 맞추 
기 위한 방법이 좀 더 보완되어야 할 것으로 생각된다. 본 연구에 서는 인공와우를 기준으로 하였을 때 인공와우 음량보다는 다소 작지만 적당하게 균형을 이루는 소리크기를 기준으로 보청기를 조절하였으며, 이러한 방법은 바이모달 착용자의 일반적인 소리 크기 균형을 맞추기 위한 방법 중 하나로 적용 가능할 것으로 생 각된다. 그러나 어느 정도가 적당한 균형을 이루는 것인지에 대 한 근거는 주관적 판단에 의존하기 때문에 주관적 판단을 가이 드할 지침이 마련되어야 할 것이다.

바이모달 결과 혜택 측정과 관련하여 주관적 설문지와 소 음하 평가가 향후 보완되어야 한다. 본 연구 결과와 Cheng et al.(2018)의 연구처럼 천장효과의 제약을 보완하기 위한 소음 하 말지각 평가가 시행되어야 한다. 또한 주관적 설문검사를 통해 바이모달 혜택의 측정이 필요한데, 다른 여러 선행 연구 (Ching et al., 2004; Cuda et al., 2019; English et al., 2016; Keilmann et al., 2009; Veugen et al., 2016a; Veugen et al., 2016b; Vroegop et al., 2019)에서도 주관적 설문지를 시 행하고 있다. 설문평가는 바이모달 보청기적합이나 추후 재활 중 재 결정에 중요한 단서를 제공할 수 있을 것이다.

본 연구를 통해 바이모달 착용 아동의 청력, 단어인지도 결과, 보청기적합에 대한 내용을 검토하였고 바이모달 아동은 청력과 단어인지도에서 향상을 보였고 보청기적합에서 실이측정을 활 용하고 있음을 확인하였다. 소음하검사, 사영역검사, 소리크기 균형검사, 바이모달 혜택을 파악하는 설문지와 배경정보 파악을 위한 설문지가 추가된다면 바이모달 아동의 보청기적합 향상과 결과 측정에 좀 더 도움이 될 수 있을 것이다. 향후 이러한 사항 을 고려하여 좀 더 체계적인 바이모달 적합이 이루어지기를 기대 한다.

중심 단어: 바이모달적합, 바이모달청각, 보청기적합, 바이모 달 아동.

\section{Ethical Statement}

$\mathrm{N} / \mathrm{A}$

\section{Acknowledgments}

$\mathrm{N} / \mathrm{A}$

\section{Declaration of Conflicting Interests}

There are no conflict of interests.

\section{Funding}

This work was supported by the Ministry of Education of the Republic of Korea and the National Research Foundation of
Korea (2019S1A5A 8038153).

\section{Author Contributions}

Conceptualization: Hyekyoung Hwang, Soo Hee Oh. Data curation: Soo Hee Oh. Investigation: Hyekyoung Hwang, Timothy Kim, Leehwa Park, Soo Hee Oh. Validation: Hyekyoung Hwang, Timothy Kim, Leehwa Park, Soo Hee Oh. Writingoriginal draft: Hyekyoung Hwang, Soo Hee Oh. Writingreview \& editing: Hyekyoung Hwang, Soo Hee Oh. Approval of final manuscript: Hyekyoung Hwang, Soo Hee Oh.

\section{ORCIDiD}

Hyekyoung Hwang https://orcid.org/0000-0001-5506-2311

Timothy Kim https://orcid.org/0000-0002-9947-2344

Leehwa Park https://orcid.org/0000-0002-1147-6333

Soo Hee Oh https://orcid.org/0000-0002-3745-1484

\section{REFERENCES}

Akeroyd, M. A. (2006). The psychoacoustics of binaural hearing. International Journal of Audiology, 45 Suppl 1, S25-S33.

Bagatto, M., Moodie, S., Scollie, S., Seewald, R., Moodie, S., Pumford, J., et al. (2005). Clinical protocols for hearing instrument fitting in the desired sensation level method. Trends in Amplification, 9(4), 199226.

Brown, C. A. \& Bacon, S. P. (2009). Achieving electric-acoustic benefit with a modulated tone. Ear and Hearing, 30(5), 489-493.

Brown, K. D. \& Balkany, T. J. (2007). Benefits of bilateral cochlear implantation: a review. Current Opinion in Otolaryngology \& Head and Neck Surgery, 15(5), 315-318.

Byrne, D. \& Noble, W. (1998). Optimizing sound localization with hearing aids. Trends in Amplification, 3(2), 51-73.

Carlson, M. L., Sladen, D. P., Haynes, D. S., Driscoll, C. L., DeJong, M. D., Erickson, H. C., et al. (2015). Evidence for the expansion of pediatric cochlear implant candidacy. Otology and Neurotology, 36(1), 43-50.

Cheng, X., Liu, Y., Wang, B., Yuan, Y., Galvin, J. J., 3rd, Fu, Q. J., et al. (2018). The benefits of residual hair cell function for speech and music perception in pediatric bimodal cochlear implant listeners. Neural Plasticity, 2018, 4610592.

Ching, T. Y., Incerti, P., \& Hill, M. (2004). Binaural benefits for adults who use hearing aids and cochlear implants in opposite ears. Ear and Hearing, 25(1), 9-21.

Crew, J. D., Galvin, J. J., 3rd, Landsberger, D. M., \& Fu, Q. J. (2015). Contributions of electric and acoustic hearing to bimodal speech and music perception. PLoS One, 10(3), e0120279.

Cuda, D., Murri, A., Mainardi, A., \& Chalupper, J. (2019). Effectiveness and efficiency of a dedicated bimodal fitting formula. Audiology Research, 9(1), 219.

Dawes, P., Munro, K. J., Kalluri, S., \& Edwards, B. (2013). Unilateral and bilateral hearing aids, spatial release from masking and auditory 
acclimatization. The Journal of the Acoustical Society of America, 134(1), 596-606.

Dorman, M. F., Gifford, R. H., Spahr, A. J., \& McKarns, S. A. (2008). The benefits of combining acoustic and electric stimulation for the recognition of speech, voice and melodies. Audiology and Neurootology, 13(2), 105-112.

D’Onofrio, K. L. \& Gifford, R. H. (2021). Bimodal benefit for music perception: effect of acoustic bandwidth. Journal of Speech, Language, and Hearing Research, 64(4), 1341-1353.

English, R., Plant, K., Maciejczyk, M., \& Cowan, R. (2016). Fitting recommendations and clinical benefit associated with use of the NAL-NL2 hearing-aid prescription in nucleus cochlear implant recipients. International Journal of Audiology, 55 Suppl 2, S45-S50.

Gifford, R. H., Dorman, M. F., Shallop, J. K., \& Sydlowski, S. A. (2010). Evidence for the expansion of adult cochlear implant candidacy. Ear and Hearing, 31(2), 186-194.

Harris, M. S. \& Hay-McCutcheon, M. (2010). An analysis of hearing aid fittings in adults using cochlear implants and contralateral hearing aids. The Laryngoscope, 120(12), 2484-2488.

Holube, I., Fredelake, S., Vlaming, M., \& Kollmeier, B. (2010). Development and analysis of an international speech test signal (ISTS). International Journal of Audiology, 49(12), 891-903.

Keilmann, A. M., Bohnert, A. M., Gosepath, J., \& Mann, W. J. (2009). Cochlear implant and hearing aid: a new approach to optimizing the fitting in this bimodal situation. European Archives of OtoRhino-Laryngology, 266(12), 1879-1884.

Kong, Y. Y. \& Carlyon, R. P. (2007). Improved speech recognition in noise in simulated binaurally combined acoustic and electric stimulation. The Journal of the Acoustical Society of America, 121(6), 3717-3727.

Kong, Y. Y., Stickney, G. S., \& Zeng, F. G. (2005). Speech and melody recognition in binaurally combined acoustic and electric hearing. The Journal of the Acoustical Society of America, 117(3 Pt 1), 13511361.

Lotfi, Y., Hasanalifard, M., Moossavi, A., Bakhshi, E., \& Ajaloueyan, M. (2019). Binaural hearing advantages for children with bimodal fitting. International Journal of Pediatric Otorhinolaryngology, 121, 58-63.

Moberly, A. C., Lowenstein, J. H., \& Nittrouer, S. (2016). Early bimodal stimulation benefits language acquisition for children with cochlear implants. Otology and Neurotology, 37(1), 24-30.

Moore, B. C. \& Malicka, A. N. (2013). Cochlear dead regions in adults and children: diagnosis and clinical implications. Seminars in Hearing, 34(1), 037-050.
Nilakantan, A., Raj, P., Saini, S., \& Mittal, R. (2018). Early speech perception test outcome in children with severe sensorineural hearing loss with unilateral cochlear implants alone versus bimodal stimulation. Indian Journal of Otolaryngology and Head and Neck Surgery, 70(3), 398-404.

Park, L. \& Oh, S. H. (2021). Speech and sound quality recognition in adults bimodal cochlear implant listeners. Audiology and Speech Research, 17(2), 198-205.

Scherf, F. W. \& Arnold, L. P. (2014). Exploring the clinical approach to the bimodal fitting of hearing aids and cochlear implants: results of an international survey. Acta Oto-laryngologica, 134(11), 1151-1157.

Shirvani, S., Jafari, Z., Motasaddi Zarandi, M., Jalaie, S., Mohagheghi, H., \& Tale, M. R. (2015). Emotional perception of music in children with bimodal fitting and unilateral cochlear implant. The Annals of Otology, Rhinology, and Laryngology, 125(6), 470-477.

Siburt, H. W. \& Holmes, A. E. (2015). Bimodal programming: a survey of current clinical practice. American Journal of Audiology, 24(2), 243-249.

Veugen, L. C., Chalupper, J., Snik, A. F., Opstal, A. J., \& Mens, L. H. (2016a). Matching automatic gain control across devices in bimodal cochlear implant users. Ear and Hearing, 37(3), 260-270.

Veugen, L. C., Chalupper, J., Snik, A. F., van Opstal, A. J., \& Mens, L. H. (2016b). Frequency-dependent loudness balancing in bimodal cochlear implant users. Acta Oto-laryngologica, 136(8), 775-781.

Vroegop, J. L., Goedegebure, A., \& van der Schroeff, M. P. (2018). How to optimally fit a hearing aid for bimodal cochlear implant users: a systematic review. Ear and Hearing, 39(6), 1039-1045.

Vroegop, J. L., Homans, N. C., van der Schroeff, M. P., \& Goedegebure, A. (2019). Comparing two hearing aid fitting algorithms for bimodal cochlear implant users. Ear and Hearing, 40(1), 98-106.

Warren, S. E. \& Dunbar, M. N. (2018). Bimodal hearing in individuals with severe-to-profound hearing loss: benefits, challenges, and management. Seminars in Hearing, 39(4), 405-413.

Yehudai, N., Shpak, T., Most, T., \& Luntz, M. (2013). Functional status of hearing aids in bilateral-bimodal users. Otology and Neurotology, 34(4), 675-681.

Yoon, Y. S., Shin, Y. R., Gho, J. S., \& Fu, Q. J. (2015). Bimodal benefit depends on the performance difference between a cochlear implant and a hearing aid. Cochlear Implants International, 16(3), 159-167.

Zhang, T., Dorman, M. F., Gifford, R., \& Moore, B. C. (2014). Cochlear dead regions constrain the benefit of combining acoustic stimulation with electric stimulation. Ear and Hearing, 35(4), 410417. 